\title{
QUALIDADE DO PÊSSEGO, cv. MACIEL, EM FUNÇÃO DE ADUBAÇÃO DE BASE MAIS FOLIAR
}

\author{
THE INFLUENCE OF BASE PLUS LEAF FERTILIZATION ON PEACH QUALITY \\ CULTIVAR 'MACIEL'
}

\author{
Alexandre GAZOLLA NETO1 \\ Clevison Luiz GIACOBBO² \\ Dalcionei PAZZIN ${ }^{1}$ \\ José Carlos FACHINELLO ${ }^{3}$
}

\begin{abstract}
RESUMO
O trabalho teve por objetivo avaliar o efeito da adubação de base mais foliar, na qualidade e produtividade pré e póscolheita de pêssegos cultivar Maciel. O experimento foi realizado em Pelotas/RS, safra 2005/06. O pomar foi conduzido com densidade de 1667 plantas ha"-1 e na forma de "Y", sem irrigação. Os tratamentos foram compostos por adubos de base com o formulado contendo nitrogênio nítrico 15,5\% + cálcio $19 \%$ + boro $0,3 \%$ e foliar com $0,5 \%$ de Kristasol $\circledast 12 \% \mathrm{~N}+45 \% \mathrm{~K}_{2} \mathrm{O}+1,2 \% \mathrm{~S}$ (Nitrogênio Nítrico e Potássio solúvel sem cloro)], em 26/08/2005 e 26/09/2005, e o formulado contendo nitrogênio nítrico $12 \%$ + potássio $12 \%$ + cálcio solúvel 15\% + boro 0,24\% (formulações 12-00-12 e 10-00-15, respectivamente) e foliar com 0,5\% da formulação contendo nitrogênio nítrico 15,5\% + cálcio solúvel em água 19\%, ambas em 20/10 e 20/11/2005. Os tratamentos foram: 1 - 0 (testemunha); 2

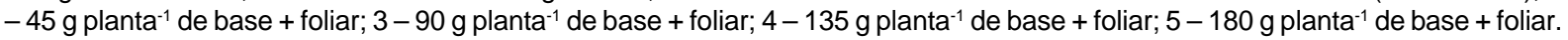
As variáveis analisadas foram firmeza de polpa, sólidos solúveis totais (SST), colorimetria, matéria fresca e seca de frutas, massa média de fruta, produtividade, classificação comercial e incidência de podridão, na colheita e no armazenamento. Nessas condições, as adubações com os formulados não apresentaram melhorias de qualidade de frutas e produtividade do pomar de pessegueiro, cv. Maciel. Verificou-se diferenças estatísticas, somente na variável armazenamento de frutas em câmera fria, onde os tratamentos $1 \mathrm{e}$ 2 aos 30 dias apresentaram menor percentagem de podridões nas frutas, diferindo do tratamento 5 . As demais variáveis não diferiram significativamente.

Palavras-chaves: Pessegueiro; nutrição; armazenamento.
\end{abstract}

The present research aimed to evaluate base plus leaf fertilization on pre and post-harvest quality and productivity of peaches cultivar 'Maciel'. The work was carried out in Pelotas/Brazil, 2005/06 crop. The tree planting density was at 1667 trees/ha in "Y" system. No irrigation method was used. The treatments consisted of 2 batches. The first one was applied on 26/08/2005 and 26/ $09 / 2005$ and consisted of base fertilization containing $15.5 \%$ nitrate $+19 \%$ calcium $+0.3 \%$ boron, and leaf fertilization containing $0.5 \%$ of Kristaso $^{\otimes}\left(12 \% \mathrm{~N}+45 \% \mathrm{~K}_{2} \mathrm{O}+1,2 \% \mathrm{~S}\right.$ (nitric nitrogen and soluble non-chloride Potassium)). The second batch was applied on $20 / 10 / 2005$ and $20 / 11 / 2005$ consisting of $12 \%$ nitrate $+12 \%$ potassium $+15 \%$ soluble calcium $+0.24 \%$ boron $(12-00-12$ and 10 00-15 fertilization ratio, respectively) for base fertilization and $0.5 \%$ of the formulation containing $15.5 \%$ nitrogen $+19 \%$ soluble calcium for leaf fertilization. The base and leaf fertilization applied for each treatment were: 1) no fertilization (control); 2$) 45 \mathrm{~g}$ tree ${ }^{-1}$; 3) $90 \mathrm{~g} \mathrm{tree}^{-1}$;4) $135 \mathrm{~g}_{\text {tree }}^{-1}$ and 5) $180 \mathrm{~g} \mathrm{tree}^{-1}$. The variables assessed were pulp firmness, total soluble solids (SST), colorimeter, fresh and dry matter of fruits, average fruit weight, yield rates, commercial ranking and decay incidence at the time of harvest and storage. The described fertilization rates did not show any improvement on fruit quality and productivity of the tested peach cultivar. The only variable that was found to be statically significant was fruit storage. Decay percentage was lower in fruits from treatments 1 and 2 at the $30^{\text {th }}$ day of storage, being significant with respect to treatment 5 . The others variables did not differ significatively.

Key-words: Peach tree; nutrition; storage.

\footnotetext{
${ }^{1}$ Acadêmico do curso de Agronomia/Estagiário do Setor de Fruticultura, Departamento de Fitotecnia - Universidade Federal de Pelotas/ Faculdade de Agronomia Eliseu Maciel FAEM/UFPel. Rua Andrade Neves, 1290, ap. 509, Centro, Pelotas - RS. E-mail: agazolla@gmail.com ${ }^{2}$ Eng. Agr. Dr., Prof. UERGS, Polo de Vacaria, Rua Antônio Ribeiro Branco, 1060.95200-000, Vacaria, RS. E-mail: clevison-giacobbo@uergs.edu.br ${ }^{3}$ Dr. Prof. Titular, Departamento de Fitotecnia, FAEM/UFPel. E-mail: jfachi@ufpel.tche.br
} 


\section{INTRODUÇÃO}

O pessegueiro, originário da China, pertence à família Rosácea, gênero Prunus (L.) e, dentre as espécies, Prunus persica Batsch é a mais importante. É planta típica de clima temperado, vigorosa, possui sistema radicular bem desenvolvido que explora principalmente a camada entre $0,2 \mathrm{~m}$ e $1,0 \mathrm{~m}$ de profundidade (PENTEADO, 1988).

No Brasil, o sistema mais empregado para a conservação de pêssegos in natura é o armazenamento em ar refrigerado. Neste sistema, o período seguro de estocagem de pêssegos varia de 5 a 40 dias, dependendo da cultivar, do ponto de colheita, da região de cultivo e da qualidade das câmaras frias. A perda de firmeza da polpa, a ocorrência de distúrbios fisiológicos, especialmente lanosidade e escurecimento interno e podridões têm sido relatadas como as principais alterações indesejáveis durante o armazenamento em AR.

Segundo OLIVEIRA e CALDAS (2004), a obtenção de boa produtividade e qualidade de frutas está diretamente ligada a uma nutrição balanceada. A nutrição mineral é fundamental tanto para a agricultura moderna quanto para a proteção ambiental, uma vez que altas produtividades agrícolas dependem em grande escala de fertilização com minerais.

A máxima eficiência econômica no uso de fertilizantes requer considerações quanto à probabilidade de resposta econômica. Assim, o melhor método de diagnose e de recomendação de adubação e de corretivos é aquele que prevê o uso desses insumos somente quando a resposta econômica for viável (FREIRE e MAGNANI, 1998).

Segundo BERNARDI et al. (2000), o potássio (K) e o cálcio $(\mathrm{Ca})$ competem fortemente pelos mesmos sítios de absorção e o excesso de um nutriente limita a absorção do outro, mostrando efeito de inibição competitiva. ROMBOLÀ et al. (2000), descreve o Ca como um elemento que desenvolve um papel importante ao conferir resistência à polpa, contribuindo para assegurar coesão elevada entre as células do fruto; previne, além disto, a desagregação das frutas por efeito da inibição da enzima responsável pela despolimerização das pectinas, a qual antecipa o amolecimento dos tecidos.

Em alguns casos, a presença de ânions não difundidos acaba formando sítios, como o de Donnan, no qual o cálcio vem acumulado. Desta maneira há poucas possibilidades deste cálcio juntar-se aos vasos lenhosos e somente o transporte via xilema, em direção acrópeta é importante para suprir de $\mathrm{Ca}^{2+}$ as frutas jovens, que transpiram na fase de desenvolvimento (MENGEL, 2002).

A absorção de boro pela planta favorece a síntese, transporte e acúmulo de açúcar, os processos reprodutivos, a atividade meristemática e controla a absorção e mobilidade de cálcio. Plantas frutíferas que apresentam sensibilidade mais acentuada à sua deficiência são: macieira, pereira, pessegueiro e videira (SOSA, 2005).
Uma boa alternativa para corrigir deficiências nutricionais em plantas perenes, segundo DIMENSTEIN (2004), pode ser através da adubação foliar, que já é uma realidade aceita pelos agricultores e que está crescendo em ritmo acelerado.

O nitrogênio é o elemento considerado de maior influência na produtividade do pessegueiro (ROMBOLÀ et al., 2000), por afetar diretamente o crescimento dos ramos (MATTOS et al., 1991; CAMPOS et al., 1996), o número de gemas floríferas e vegetativas (MATTOS et al.,1991) e o número de frutos por planta (MATTOS et al., 1991). Esse elemento pode propiciar um período maior de manutenção das folhas, com conseqüente aumento no período de acúmulos de reservas para o ciclo posterior (MONTE SERRAT et al., 2004). No entanto, - suprimento excessivo pode levar ao superbrotamento, provocando o sombreamento excessivo e diminuindo a insolação dos frutos (MATTOS et al., 1991).

$\mathrm{O}$ uso de $\mathrm{N}$ pode interferir ainda no aspecto qualitativo da produção, afetando o tamanho dos frutos (MATTOS et al., 1991), a coloração da casca, a firmeza da polpa e a porcentagem de sólidos solúveis totais (CAMPOS et al., 1996).

Neste contexto, o trabalho teve por objetivo avaliar o efeito da adubação de base mais foliar, na melhoria da qualidade de frutas de pêssegos, cultivar Maciel.

\section{MATERIAL E MÉTODOS}

O experimento foi conduzido em Pelotas/RS, situado na latitude $31^{\circ} 52^{\prime} 00^{\prime \prime} \mathrm{S}$, longitude $52^{\circ} 21^{\prime}$ 24" W Greenwich e altitude de 13,24 m, durante o período de 26 de agosto a 26 de novembro de 2005. O solo pertence à unidade de mapeamento Camaquã, sendo classificado como Argisolo Vermelho Amarelo (EMBRAPA, 2006), em plantas de pessegueiro, com cinco anos de idade da cultivar Maciel, frutas de dupla finalidade na safra 2005/06

O pomar é de média alta densidade de plantio, com 1667 plantas ha ${ }^{-1}$ e as plantas conduzidas em forma de " $Y$ ", sem irrigação, com cobertura vegetal de aveia preta, sendo acamada no período de floração das plantas de pessegueiro.

No inicio do experimento as plantas estavam em plena floração. Os Tratamentos foram compostos por adubo de base com o formulado contendo nitrogênio nítrico $15,5 \%$ + cálcio $19 \%$ + boro $0,3 \%$, em 26/08/2005 e 26/09/2005 + foliar com 0,5\% de Kristasol® $\left[12 \% \mathrm{~N}+45 \% \mathrm{~K}_{2} \mathrm{O}+1,2 \% \mathrm{~S}\right.$ (Nitrogênio Nítrico e Potássio solúvel sem cloro)], e o formulado contendo nitrogênio nítrico $12 \%$ + potássio $12 \%$ + cálcio solúvel $15 \%$ + boro $0,24 \%$, em $20 / 10$ e $20 / 11 /$ 2005 (formulações 12-00-12 e 10-00-15, respectivamente) + foliar com $0,5 \%$ da formulação contendo nitrogênio nítrico $15,5 \%$ + cálcio solúvel em água $19 \%$. Os tratamentos foram: 1 - 0 g planta${ }^{1}$ (testemunha); 2 - 45 g planta $^{-1}$ de base + foliar; 3 $90 \mathrm{~g}$ planta $^{-1}$ de base + foliar; $4-135 \mathrm{~g}^{-1 a n t a}{ }^{-1}$ de base + foliar; 5 - $180 \mathrm{~g}$ planta $^{-1}$ de base + foliar.

As demais práticas culturais foram seguidas normalmente e de maneira homogênea para todos 
os tratamentos, conforme recomendado para a cultura. A colheita foi realizada em várias etapas, conforme os frutos atingiam o ponto de maturação. O raleio foi realizado 30 dias após a queda das pétalas. Nesta época os frutos apresentavam diâmetro entre 1,5 a $2 \mathrm{~cm}$. Foram deixados um fruto a cada 5 a $8 \mathrm{~cm}$ e, nos raminhos finos, apenas um fruto. Com relação à análise química do solo na área do experimento, esta foi realizada antes da instalação do experimento, na projeção da copa, de acordo com a Tabela 1.

TABELA 1 - Resultado da análise química do solo, na projeção da copa entre 0-20 cm de profundidade, realizada em 2005, na data da instalação do experimento (Pelotas- RS).

\begin{tabular}{|c|c|c|c|c|c|c|c|c|c|}
\hline \multirow{2}{*}{$\begin{array}{l}\text { Argila } \\
\mathrm{g} \mathrm{kg}^{-1}\end{array}$} & \multirow{2}{*}{$\begin{array}{c}\mathrm{pH} \\
\mathrm{H}_{2} \mathrm{O}\end{array}$} & \multirow{2}{*}{$\begin{array}{c}\mathrm{pH} \\
\text { SMP }\end{array}$} & \multirow{2}{*}{$\begin{array}{c}\text { M.O. } \\
\%\end{array}$} & \multirow{2}{*}{\multicolumn{3}{|c|}{ 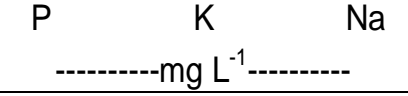 }} & $\mathrm{Al}$ & $\mathrm{Ca}$ & $\mathrm{Mg}$ \\
\hline & & & & & & & \multicolumn{3}{|c|}{$-\mathrm{Cmol}_{\mathrm{C}} \mathrm{L}^{-1}$} \\
\hline 200 & 6,0 & 6,5 & 1,9 & 23,0 & 102,0 & 20,0 & 0,0 & 5,8 & 2,0 \\
\hline
\end{tabular}

O delineamento estatístico utilizado foi de blocos ao acaso, com três repetições por tratamento e três plantas por repetição, onde avaliou-se a firmeza de polpa, sólidos solúveis totais (SST), colorimetria (coloração da epiderme), matéria fresca e seca de frutos, massa média do fruto, produtividade, classificação comercial dos frutos de acordo com o tamanho e incidência de podridão no momento da colheita e em câmara fria, pós colheita.

A firmeza da polpa $(\mathrm{N})$ foi realizada com penetrômetro manual, através de duas leituras na região equatorial dos frutos em lados diametralmente opostos, e após a remoção da epiderme. O SST (Brix) pela leitura direta em refratômetro manual, marca Atago N1, com correção da temperatura. A coloração da epiderme dos frutos foi realizada em doze frutos por tratamento e determinada mediante duas leituras em lados opostos e no sentido equatorial, sendo os respectivos valores obtidos em coordenadas padrão C.I.E. L* $a^{*} b^{*}$ e convertidos para ângulo Hue $\left({ }^{\circ} h^{*}=\operatorname{tang}^{*-1} b^{*} \cdot a^{*-1}\right)$, com uso de colorímetro Minolta ${ }^{\circledR}$ CR-300 com fonte de luz D 65 , com ponteiro para emissão de facho de luz de $8 \mathrm{~mm}$ de abertura. A matéria fresca foi determinada através da massa de quatro frutos recém colhidos, os quais após terem sido pesados foram levados para estufa a uma temperatura de $65^{\circ} \mathrm{C}$ até secagem completa e em seguida foi pesado novamente para determinação da matéria seca. A massa média dos frutos, realizado através de balança de precisão.

A produtividade estimada foi determinada através da produção total obtida em $\mathrm{kg}$ na parcela e estimada para um hectare e transformada para t ha 1. A classificação das frutas foi realizada com a quantidade total de frutas colhidas, classificando-as em porcentagem de; tipo 1 - com diâmetro maior ou igual a $57 \mathrm{~mm}$, tipo $2-$ de 47 a $57 \mathrm{~mm}$ e tipo $3-$ de
44 a $47 \mathrm{~mm}$. As podridões no momento da colheita foram avaliadas segundo o número de frutas com presença de podridões, determinados em porcentagem e no armazenamento foram determinadas através do acompanhamento, em câmara fria, de 10 frutas de cada tratamento, os quais foram acondicionados em redes e armazenados em câmara fria com temperatura de $2{ }^{\circ} \mathrm{C} \pm 2$, e as avaliações realizadas aos 20,30, 37 e 42 dias após a armazenagem, avaliando-se o número de frutas com podridões.

Os dados foram submetidos à análise da variância pelo teste $\mathrm{F}$ e comparados entre si pelo teste de Duncan ao nível de $5 \%$ de significância. Os dados expressos em porcentagem (frutas podres na colheita, tipificação e podridões pós-colheita) foram transformados em arco seno da raiz de $x / 100$. A análise foi realizada através do programa estatístico WinStat (MACHADO e CONCEIÇÃO, 2002).

\section{RESULTADOS E DISCUSSÃO}

De acordo com os resultados obtidos, verificou-se diferenças significativas apenas para a variável podridões de frutos pós-colheita, aos 30 dias após armazenados em câmara fria. Nas demais variáveis não foram verificadas diferenças, conforme Tabelas 2 e 3, demonstrando assim que a adubação suplementar, praticamente, não influenciou na qualidade de frutas e produtividade do pessegueiro cultivar Maciel. Indicando que as reservas das plantas e/ou as quantidades fornecidas pelo solo estavam em condições normais para proporcionar adequado desenvolvimento e produtividade das plantas. 
GAZOLLA NETO, A. et al. Qualidade do pêssego...

TABELA 2 - Firmeza da polpa, colorimetria, matéria fresca (MF) e seca (MS), produtividade e podridão nas frutas em pessegueiro, cv. Maciel, em função de diferentes dosagens de adubação de base e da adubação foliar (Pelotas, FAEM/UFPel, 2006).

\begin{tabular}{ccccccc}
\hline Trat. & Firmeza (N) & $\begin{array}{c}\text { Colorimetria } \\
\left({ }^{\circ H u e}\right)\end{array}$ & $\begin{array}{c}\text { MF } \\
(\mathrm{g})\end{array}$ & $\begin{array}{c}\text { MS } \\
(\mathrm{g})\end{array}$ & Produtividade $\left(\mathrm{t} \mathrm{ha}{ }^{-1}\right)$ & $\begin{array}{c}\text { Frutas } \\
\text { podres } \\
(\%)\end{array}$ \\
\hline T1 $-0 \mathrm{~g}$ & $85,12^{\text {ns }}$ & $88,31^{\text {ns }}$ & $590,38^{\text {ns }}$ & $98,82^{\text {ns }}$ & $15,75^{\text {ns }}$ & $18,01^{\text {ns }}$ \\
T2 - $45 \mathrm{~g}$ & 79,63 & 87,45 & 590,76 & 105,55 & 15,51 & 11,40 \\
T3-90 g & 82,28 & 87,78 & 543,27 & 91,34 & 17,16 & 15,68 \\
T4 - 135 g & 87,87 & 89,20 & 575,63 & 95,73 & 13,32 & 15,85 \\
T5 - 180 g & 84,44 & 88,79 & 549,43 & 95,68 & 15,38 & 16,55 \\
\hline C.V. & 20,25 & 5,71 & 7,66 & 6,97 & 40,35 & 33,72 \\
\hline
\end{tabular}

ns. Não significativo pelo teste de Duncan a $5 \%$ de significância.

TABELA 3 - Classificação e podridão de frutos, de pêssegos, cv. Maciel, em função de diferentes dosagens de adubação de base e foliar e armazenagem em câmara fria (Pelotas, FAEM/UFPel, 2006).

\begin{tabular}{|c|c|c|c|c|c|c|c|}
\hline \multirow{2}{*}{ Trat. } & \multicolumn{3}{|c|}{ Classificação (\%) } & \multicolumn{4}{|c|}{ Podridão em câmara fria (\%) } \\
\hline & Tipo 1 & Tipo 2 & Tipo 3 & 20 dias $^{*}$ & 30 dias $^{*}$ & 37 dias $^{*}$ & 42 dias $^{*}$ \\
\hline $\mathrm{T} 1-0 \mathrm{~g}$ & $78,30^{\text {ns }}$ & $19,62^{\text {ns }}$ & $2,07^{\mathrm{ns}}$ & $0,00 \mathrm{aB}$ & $0,00 \mathrm{bB}$ & 23,33 aA & $16,67 \mathrm{abAB}$ \\
\hline $\mathrm{T} 2-45 \mathrm{~g}$ & 79,40 & 18,17 & 2,42 & $0,00 \mathrm{aB}$ & $0,00 \mathrm{bB}$ & $10,00 \mathrm{aAB}$ & $20,00 a b A$ \\
\hline $\mathrm{T} 3-90 \mathrm{~g}$ & 75,52 & 19,68 & 4,79 & $0,00 \mathrm{aB}$ & $13,33 a b A B$ & $30,00 \mathrm{aA}$ & 3,33 bB \\
\hline $\mathrm{T} 4-135 \mathrm{~g}$ & 77,33 & 20,10 & 2,57 & $3,33 \mathrm{aA}$ & $16,34 \mathrm{abA}$ & $10,00 \mathrm{aA}$ & $16,67 \mathrm{abA}$ \\
\hline $\mathrm{T} 5-180 \mathrm{~g}$ & 75,49 & 22,42 & 2,09 & $0,00 \mathrm{aB}$ & 26,67 aA & $16,67 \mathrm{aAB}$ & 26,67 aA \\
\hline C.V. \% & 13,20 & 27,09 & 101,82 & 387,30 & 106,62 & 82,32 & 65,63 \\
\hline
\end{tabular}

Letras distintas, minúscula na coluna e maiúscula na linha, diferem significativamente entre si pelo teste de Duncan a $5 \%$ de significância.

ns. Não significativo pelo teste de Duncan a $5 \%$ de significância.

* Dias após acondicionado em câmara fria.

A baixa produtividade do pomar, esta aliada à ocorrência de geada no período de floração, o que acarretou uma significativa perda de flores, baixando a produtividade do pomar.

Em relação ao cálcio e boro, aplicados via foliar, os resultados são contraditórios, enquanto existem autores que citam efeitos positivos como é o caso de PLICH e WÓJCIK (2002), os quais estudaram durante três anos a aplicação foliar de cálcio, em duas cvs. de ameixeira Stanley e Dabrowicka e verificaram que a aplicação de cálcio causou aumento significativo na firmeza de polpa na cv. Stanley, no momento da colheita e conseqüentemente apresentou melhor resistência durante o período de armazenamento.

Normalmente, o pêssego não resiste a um período superior a quatro semanas de armazenamento. Neste caso as avaliações feitas aos 42 dias de armazenamento, em função dos frutos apresentarem murchamento, realizou-se a última avaliação e descarte das frutas restantes, mesmo não apresentando podridões.

Com relação à incidência de podridões nas frutas armazenados em câmara fria com $2{ }^{\circ} \mathrm{C} \pm 2$, verificou-se, conforme dados apresentados na Tabela 3 , que aos 30 e 42 dias de armazenagem, no tratamento cinco houve influência negativa da adubação nitrogenada, aumentando o percentual de frutas com podridão em relação à testemunha, indicando que as quantidades de nitrogênio acumuladas do ano anterior e aquelas fornecidas pelo solo foram suficientes. Estes dados também foram obtidos por GIACOBBO et al. (2006), em estudo sobre a qualidade do pêssego, cv. Eldorado em função da adubação, os mesmos, constataram que a adubação com Nitrabor ${ }^{\circledR}$ melhorou a coloração das frutas, aspecto considerado importante para comercialização in natura das mesmas, porém elevou o índice de podridões nas frutas, verificou também que aplicações seguidas de Nitrabor ${ }^{\circledR}$ e Calcinit ${ }^{\circledR}+$ Krista ${ }^{\circledR}$ K 45S, não aumentaram a produtividade do 
pomar. Estes resultados estão de acordo com o relato de ROMBOLÀ et al. (2000), o excesso de nitrogênio tem uma forte relação com a incidência de podridões nas frutas.

Quando comparado as diferentes datas para cada tratamento, verificou-se, no geral que os maiores incidências de podridões se apresentaram aos 30 e 42 dias, com destaque para o tratamento contendo $90 \mathrm{~g} \mathrm{planta}^{-1}$ de adubação de base + foliar que apresentou o pico máximo de podridões aos 37 dias.

\section{CONCLUSÕES}

Nas condições em que foi realizado o trabalho, as adubações com os formulados contendo nitrogênio nítrico $15,5 \%$ + cálcio $19 \%$ + boro $0,3 \%$ + foliar com $0,5 \%$ de Kristasol® $\left(12 \% \mathrm{~N}+45 \% \mathrm{~K}_{2} \mathrm{O}+\right.$ $1,2 \%$ S (Nitrogênio Nítrico e Potássio solúvel sem cloro)) e nitrogênio nítrico $12 \%$ + potássio $12 \%$ + cálcio solúvel $15 \%$ + boro $0,24 \%$ + foliar com $0,5 \%$ da formulação contendo nitrogênio nítrico 15,5\% + cálcio solúvel em água 19\%, não apresentaram melhorias de qualidade de frutas e produtividade do pomar de pessegueiro, cv. Maciel.

Não houve incidência de podridões até os 30 dias de armazenamento nos tratamentos com ausência de adubos ou quando utilizou-se a dose de $45 \mathrm{~g}$.

\section{REFERÊNCIAS}

1. BERNARDI, A.C. de C.; CARMELLO, Q.A. de C.; CARVALHO, S.A. de. Macronutrientes em mudas de citros cultivadas em vasos em resposta à adubação NPK. Scientia Agricola, v. 57, n. 4, p. 761-767, 2000.

2. CAMPOS, A.D.; FREIRE, C.J.S.; NAKASU, B.H.; FORTESW, J.F. Qualidade dos frutos e crescimento dos ramos de pessegueiro em função do nitrogênio e potássio foliar. In: CONGRESSO BRASILEIRO DE FRUTICULTURA, 14., , 1996, Curitiba. Anais. Curitiba, SBF, 1996. p. 379.

3. DIMENSTEIN, L. Nutrição foliar em fruteiras. In.: ENCONTRO NACIONAL SOBRE FRUTICULTURA DE CLIMA TEMPERADO - ENFRUTE, 7., 2004, Fraiburgo. Anais. Caçador: Epagri, 2004. p. 9-14.

4. EMBRAPA. Sistema Brasileiro de Classificação de Solos. 2. ed. Rio de Janeiro: Embrapa Solos, 2006. $306 \mathrm{p}$.

5. FREIRE, C.J. da S.; MAGNANI, M. Adubação e correção do solo. In: A cultura do pessegueiro. MEDEIROS, C.A.B.; RASEIRA, M. do C.B. (Eds.). Brasília: Embrapa -SPI; Pelotas: Embrapa-CPACT, 1998. p. 161-187.

6. GIACOBBO, C.L.; PICOLOTTO, L.; KRÜGER, L.R.; PEREIRA, I. dos S.; ZUCHI, J.; FACHINELLO, J.C.. Influenza della fertilizzazione sulla qualità della pesca (cv Eldorado). Italus Hortus e Notiziario Soi Di Ortoflorofrutticoltura, v. 13, n. 3, p. 94-97, 2006.

7. MACHADO, A.; CONCEIÇÃO, A.R. Programa estatístico WinStat - Sistema de Análise Estatístico para Windows, versão 2.0. Pelotas, 2002.

8. MATTOS, M.L.T.; FREIRE, C.J.S.; MAGNANI, M. Produção do pessegueiro cv. Diamante, sob diferentes doses de nitrogênio aplicado ao solo. Pesquisa Agropecuária Brasileira, v. 26, p. 113-117, 1991.

9. MENGEL K. Alternative or complementary role of foliar supply in mineral nutrition. Proceeding of the international symposium on foliar nutrition of perennal fruit plants. Acta Horticulturae, n. 594, p. 33-47, 2002.

10. MONTE SERRAT, B.; REISMANN, C.B; MOTTA, A.C.V.; MARQUES, R. Nutrição mineral de fruteira de caroço. In: MONTEIRO, L.B.; MAY DE MIO, L.L.; MONTE SERRAT, B.; MOTTA A.C.; CUQUEL F.L. Fruteiras de caroço: uma visão ecológica, Curitiba: UFPR, 2004. p. 71-95.

11. OLIVEIRA, A.M.G.; CALDAS, R.C. Produção do mamoeiro em função de adubação com nitrogênio, fósforo e potássio. Revista Brasileira Fruticultura, v. 26, n. 1, p. 160-163, 2004.

12. PENTEADO, S.R. Fruticultura de clima temperado em São Paulo. Campinas: Fundação Cargill, 1988. p. 55-90.

13. PLICH, H.; WÓJCIK, P. The effect of calcium and boron foliar application on postharvest plum fruit quality. Acta Horticulturae, n. 594, p. 445-451, 2002.

14. ROMBOLÀ, A.D.; TOSELLI, M.; SCUDELARI, D.; TAGLIAVINI, M.; MARANGONI, B. A nutrição das frutas de caroço na fruticultura eco-compatível. In: SIMPÓSIO INTERNACIONAL DE FRUTAS DE CAROÇO, 2000, Porto Alegre. Anais. Porto Alegre, 2000. p. 41-60.

15. SOSA, D.H. El boro en los frutales del género Prunus, Pyrus y Malus. Artículos Técnicos do Instituto de Transferencia de Tecnología - ACE. Alto Valle de Río Negro y Neuquén. Disponível em: <http://www.intecace.com.ar/articulos/boro.htm>. Acesso em 26 mar de 2005.

Recebido em 22/12/2006 Aceito em 06/08/2007 
